\title{
A Self-Adaptive Image Processing Application Based on Evolvable and Scalable Hardware
}

\author{
Ángel Gallego, Javier Mora, Andrés Otero, Blanca López, Eduardo de la Torre, Teresa Riesgo
}

Evolvable Hardware (EH) is a technique that consists of using reconfigurable hardware devices whose configuration is controlled by an Evolutionary Algorithm (EA). Our system consists of a fully-FPGA implemented scalable EH platform, where the Reconfigurable processing Core (RC) can adaptively increase or decrease in size. Figure 1 shows the architecture of the proposed System-on-Programmable-Chip (SoPC), consisting of a MicroBlaze processor responsible of controlling the whole system operation, a Reconfiguration Engine (RE), and a Reconfigurable processing Core which is able to change its size in both height and width. This system is used to implement image filters, which are generated autonomously thanks to the evolutionary process. The system is complemented with a camera that enables the usage of the platform for real time applications.

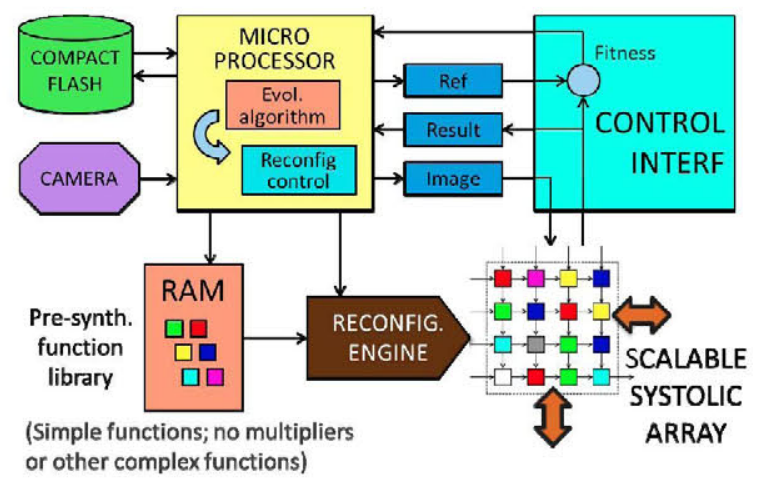

Figure 1. Overview of the System Architecture

The $\mathrm{RC}$ architecture is based on a two dimensional meshtype systolic array of Processing Elements (PEs). Each PE is a basic computational unit (occupying just 5 CLBs) able to perform a single operation per clock cycle, with the data taken from their close neighbors. Each PE includes a Functional Block that performs a basic operation with one or two input signals (North and West), and sends the result through two outputs (South and East). With DPR, the PEs can be changed at runtime, and as an extension of previous systems shown in [1], also the surrounding elements (input multiplexors and output selector) are reconfigurable. This allows the array to scale up or down as desired, obtaining more or less processing resources according to the needs.
Another feature of the system is self-adaptivity to the different conditions in the type and level of noise, and fault tolerance due to the usage of the same hardware in both the evolution stage and the filtering stage. This fault tolerance was described in [2], showing the self-healing capability of recovering from many permanent accumulated faults. This can be achieved by injecting a fault to emulate a damaged PE.

A special usage of the real time images obtained from the camera is shown in Figure 2. The system includes a noise generator that emulates different types and levels of noise to be filtered, adding it to the images taken from the camera. With this emulator, evolution with real time images can be carried out. Furthermore, the possibility of evolving with two consecutive noisy frames is shown, obtaining results comparable to the ones obtained by traditional evolution, with the advantage of adapting to whichever conditions of noise type and level the input images have, rather than depending on previously stored training images for a pre-characterized noise type and level.

The combination of EH and scalability yields better results than any of our previously published results.

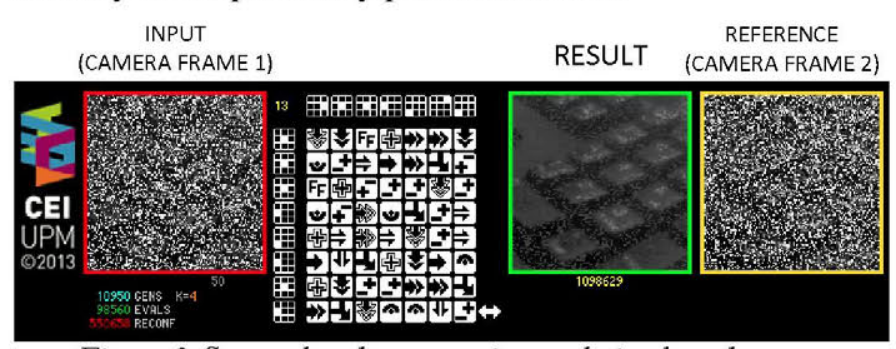

Figure 2. Screenshot demonstrating evolution based on two consecutive camera noisy frames

\section{REFERENCES}

[1] Otero, A.; Salvador, R.; Mora, J.; de la Torre, E.; Riesgo, T.; Sekanina, L.; , "A fast Reconfigurable 2D HW core architecture on FPGAs for evolvable Self-Adaptive Systems," NASA/ESA Conference on Adaptive Hardware and Systems (AHS), 2011

[2] Salvador, Ruben; Otero, Andres; Mora, Javier; Torre, Eduardo de la; Sekanina, Lukas; Riesgo, Teresa; , "Fault Tolerance Analysis and SelfHealing Strategy of Autonomous, Evolvable Hardware Systems," International Conference on Reconfigurable Computing and FPGAs (ReConFig), 2011 